\title{
Exploring Active Travel and Leisure-Time Physical Activity Relationships With Cognition Among Older Adults
}

\author{
Madhura Phansikar and Sean P. Mullen
}

\begin{abstract}
Leisure-time physical activity (LTPA) is known to benefit cognition among older adults, but the impact of active travel is unclear. To explore this relationship, data from the 2011-2014 National Health and Nutritional Examination Survey $(N=2,702$; mean age $=70$ ) were retrieved on the self-reported frequency and duration of active travel (walking/cycling for transport, $>20$ min), LTPA engagement (e.g., sport), and three cognitive outcomes. Four groups were created according to physical activity guidelines (600 metabolic equivalent of task/week): inactive $(n=1,790)$, active travelers $(n=210)$, engaging in LTPA $(n=579)$, and engaging in both $(n=123)$. Analysis of covariance (and follow-up comparisons) revealed a significant main effect for each cognition variable, after adjusting for the covariates, indicating that those engaging in LTPA performed the best. Although correlational, these findings suggest that LTPA engagement may be important for cognition among older adults, but active travel did not provide added benefit.
\end{abstract}

Keywords: active commute, active transport, executive function, exercise, memory

Engagement in physical activity is known to have cognitive benefits across the lifespan (Hillman, Erickson, \& Kramer, 2008). Among older adults, leisure-time physical activity (LTPA) has been shown to have protective effects against neurodegenerative diseases and age-related cognitive decline (Bherer, Erickson, \& LiuAmbrose, 2013; Kramer \& Erickson, 2007). In a review of crosssectional, longitudinal, and intervention studies among older adults by Bherer et al. (2013), physical activity, including structured exercise, was associated with changes in brain structure and function, a reduced risk of developing dementia and having mild cognitive impairment, and improvement in cognitive domains, such as executive functioning, memory, processing speed, and attention. For example, Rovio et al. (2005) found that those engaging in LTPA at least twice per week in midlife had lower odds of having dementia later in life. Other studies have reported that LTPA engagement is associated with improvements in memory (Richards, Hardy, \& Wadsworth, 2003) and that higher intensity LTPA is associated with better executive functioning, processing speed, memory, and overall cognitive function (Angevaren et al., 2007). Although LTPA is beneficial for improving a variety of health outcomes, it is less clear whether non-LTPA offers similar benefits.

One type of non-LTPA is known as active travel, generally described as walking or cycling to and from places for 10 or more minutes. Although active travel (also known as active commute or active transportation) involves walking or cycling that can constitute LTPA, LTPA is unique from active travel in that it is a purposeful form of leisure. While it can be done for health purposes, engagement in active travel refers solely to walking or bicycling as a means of getting from one place to another, such as work, grocery stores, etc. (Cerin, Nathan, Van Cauwenberg, Barnett, \& Barnett, 2017), whereas LTPA involves other activities, such as running or bicycling at the gym, dancing, playing sports, etc. The Sleep, Leisure,

Phansikar and Mullen are with the University of Illinois at Urbana-Champaign, Champaign, IL. Mullen is also with the Beckman Institute for Advanced Science and Technology, University of Illinois at Urbana-Champaign, Champaign, IL; and Illinois Informatics Institute, Champaign, IL. Mullen (spmullen@illinois.edu) is corresponding author.
Occupation, Transportation, and Home model for increasing physical activity describes different contexts in which one can be physically active (except sleep) and provides further support for the distinction between LTPA and active travel (Pratt, Macera, Sallis, O'Donnell, \& Frank, 2004). Furthermore, the two types of activities may differ in the extent to which they are cognitively engaging. For example, certain types of LTPA, such as martial arts and dance, are theorized to require more neuromuscular complexity than engaging in active travel (Diamond \& Ling, 2006), and LTPA may provide cognitive stimulation through continuous social interaction (e.g., engaging in group classes). Even for a common activity such as walking, engaging in walking for leisure may last longer in duration or reach higher intensity levels than active traveling. For example, active traveling is likely to be more restricted by environmental and situational factors such as climate, road traffic, required clothing for one's intended destination (e.g., heavy uniforms, business suits), or items being carried (backpack with books, food shopping bags). In terms of the length of activity, research has shown that both acute (Barella, Etnier, \& Chang, 2010) and chronic LTPAs (Weuve et al., 2004) have positive effects on cognition among older adults, and potential mechanisms for cognitive benefit range from an increase in cerebral blood flow to changes in brain structure and function (Gligoroska \& Manchevska, 2012). Among children, similar acute benefits have been found for some types of physical activities, such as simple aerobic exercise (Kamijo \& Abe, 2019; O’Leary, Ponitfex, Scudder, Brown, \& Hillman, 2011), but not for cognitively demanding exercises (Gallotta et al., 2012; Kamijo \& Abe, 2019). In this study, self-reported cross-sectional data, reflecting one's typical activity during the week, were the target of the investigation.

Although LTPA offers potentially unique benefits, active travel may be an important avenue for physical activity among older adults. It may be particularly important for older adults who reduce automobile driving (Oxley, 2015), are in retirement, and belong to lower socioeconomic groups with no access to a car (Chudyk et al., 2015). Additionally, a high amount of active travel among older adults is associated with certain demographic and environmental factors, such as a good neighborhood or physical environment (Cerin et al., 2017), 
high walkable neighborhoods (King et al., 2011), access to stores and services in the neighborhood (Chudyk et al., 2015), seasonal changes, and retiring with high incomes (Yang, Roux, \& Bingham, 2011). As of 2011 , about $15 \%$ of the older adults living in the United States engaged in at least one active travel trip/day (Yang et al., 2011). Among older adults (aged 65 years and older), the most popular destinations visited through active travel were grocery stores (Chudyk et al., 2015; Michael, Green, \& Farquhar, 2006; Smith \& Sylvestre, 2001); friends/relatives, places of recreation (Smith \& Sylvestre, 2001); and malls, cafes, and restaurants (Chudyk et al., 2015; Michael et al., 2006).

Research has shown that active travel is beneficial for physical health. For example, Vancampfort et al. (2018) examined the relationship between active travel and comorbidities among 14,585 older adults (aged 65 years and older) in six low- and middle-income countries. They found that individuals who engaged in lower levels of active travel ( $<10 \mathrm{~min} /$ day) were $28 \%$ more likely to have multimorbidity as compared to those who engaged in moderate (10-119 min/day) and high ( $>120 \mathrm{~min} /$ day) levels of active travel. Among older adults, engaging in moderate to high levels of active travel has been associated with a lower body mass index, reduced risk of having overweight, and lower systolic blood pressure (Laverty, Palladino, Lee, \& Millett, 2015).

Among older adults, the impact of active travel on cognitive aspects important for healthy aging, such as executive functioning, is less clear. Although studies have shown that walking is associated with better cognitive functioning among older adults, these studies have focused on walking as a form of LTPA (Abbott et al., 2004; Scherder et al., 2014; Yaffe, Barnes, Nevitt, Lui, \& Covinsky, 2001). For example, in a cross-sectional study of older adult women (mean age $=70$ years), Weuve et al. (2004) found that women who engaged in at least $1.5 \mathrm{hr}$ of leisure-time walking had better scores in tasks of category fluency, working memory and attention, verbal memory, global cognitive score, and the Telephone Interview for Cognitive Status compared with those who were sedentary. In terms of walking or cycling as a form of active travel, most of the studies exploring its association with cognition have been conducted among children and adolescents. For example, Van Dijk, De Groot, Van Acker, Savelberg, and Kirschner (2014) found a significant relationship between active travel to school (assessed via accelerometer) and cognitive performance on the $\mathrm{d} 2$ test of attention among girls, but no association with the Symbol Digit Modalities Test and academic achievement. Another study found that walking to school for 3060 min was associated with higher academic performance in language and mathematics (García-Hermoso, Saavedra, Olloquequi, \& Ramírez-Vélez, 2017). These studies suggest that active travel may be beneficial for certain aspects of cognition, such as academic performance and attention, among children and adolescents. Considering that older adults face age-related cognitive decline, it would be worthwhile to examine whether active travel is associated with improvements in cognition among older adults. Rovio et al. (2007) examined whether occupational physical activity and active commute to work in late midlife played a protective role against the risk of dementia in old age. The mean age of the participants when active travel was assessed was 50 years. They found no statistically significant association between active travel and dementia risk, although the data showed a trend toward a lower risk of dementia among those who engaged in a high level of active travel (60 min or more daily) and those who engaged in no active travel, compared with those who engaged in $<59$ min of active travel. In another large-scale study of older adults living in China (mean age $=69.2$ years), it was suggested that active travel may mediate the relationship between built environment and cognitive function. In this study, active travel was merely used as a covariate, which did attenuate the association between the built environment and cognitive functioning ( $\mathrm{Ng}$ et al., 2018). Another study showed that incidental physical activity, which refers to engaging in daily unstructured physical activities such as household chores and active travel, is associated with cognitive benefits in older adults (Sanchez-Lopez et al., 2018), but researchers did not isolate the impact of these activities.

The association between active travel on executive function and memory among older adults has not been rigorously studied. Interventions have been designed to increase active travel among adults (Ogilvie et al., 2007); yet, less is known about the effects of active travel on cognition. This study was designed to test whether LTPA and active travel, separately and combined, are associated with greater levels of cognitive performance in a sample of older adults. Considering active travel is an important avenue for physical activity, it is important to understand the extent to which it has cognitive benefits and whether accumulated LTPA coupled with active travel provides an added benefit. In line with prior research, we hypothesized that LTPA will have a positive association with cognition. Given the lack of consensus regarding the relationship between active travel and cognition in older adults, we had no a priori hypothesis. To explore these relationships, data from the 2011-2014 National Health and Nutritional Examination Survey (NHANES) was retrieved to examine the relationship between active travel, LTPA, and executive function and memory among older adults.

\section{Method}

\section{Sample}

NHANES is an ongoing survey examining the nutritional and health status of noninstitutionalized children and adults who reside in the United States and is administered to a nationally representative sample of 5,000 people every year. The survey assesses demographic information, dietary and health behavior, and health status (e.g., chronic conditions) for all respondents, along with cognitive assessments that are administered only to older adults (aged 60 years and older). Before beginning any assessments, written informed consent is obtained by an NHANES representative. The data used in this study are deidentified and made publicly available by NHANES. All cognitive assessments were administered in person by a trained interviewer. For the purpose of this study, data from the 2011-2012 cycle and 2013-2014 cycle of NHANES were included in the analysis. Data from these two cycles were combined and analyzed as a single data set, given that these data were cross-sectional (with different respondents in each cycle) and allowed for a larger sample size and, potentially, a more representative data set. The respondents who had complete responses on all measures (physical activity, cognition, and demographics) were included for analysis $(N=2,702)$ and had a mean age of 70 years. The demographic characteristics of the sample are provided in Table 1. The sample from 2011 to 2014 included an oversampling of minorities (Hispanics, non-Hispanic Blacks, nonHispanic Asians), lower income Whites, and older adults. More information about NHANES is available at https://www.cdc.gov/ nchs/nhanes/index.htm. This study was approved by the University of Illinois Urbana-Champaign Institutional Review Board.

\section{Measures}

Physical activity. Physical activity was assessed through selfreport via questions based on the Global Physical Activity 
Table 1 Demographic and Physical Activity Information Across the Four Groups of Physical Activity

\begin{tabular}{|c|c|c|c|c|}
\hline Demographic & Inactive & Active travel & LTPA & Both activities \\
\hline$N$ & 1,790 & 210 & 579 & 123 \\
\hline Age in years, $M(S D)$ & $70.45(6.96)$ & $68.04(6.55)$ & $69.55(6.90)$ & $67.65(6.25)$ \\
\hline Body mass index, $M(S D)$ & $25.48(7.78)$ & $25.92(7.58)$ & $25.00(7.54)$ & $23.81(6.67)$ \\
\hline Female $(\%)$ & 54.2 & 44.8 & 47.5 & 38.7 \\
\hline \multicolumn{5}{|l|}{ Race $(\%)$} \\
\hline Non-Hispanic White & 46.4 & 30.2 & 49.6 & 42.6 \\
\hline Non-Hispanic Black & 25.1 & 23.1 & 21.8 & 21.9 \\
\hline Non-Hispanic Asian & 7.6 & 13.2 & 12.6 & 18.7 \\
\hline Hispanic & 19.3 & 31 & 15.2 & 14.8 \\
\hline Other/multiracial & 1.6 & 2.5 & 1 & 2 \\
\hline \multicolumn{5}{|l|}{ Educational status (\%) } \\
\hline 11th grade or less & 32.7 & 39.8 & 16.3 & 19.3 \\
\hline High school & 24.7 & 18.6 & 20.4 & 17.4 \\
\hline Some college & 24.9 & 26.5 & 30.3 & 29 \\
\hline College graduate & 17.8 & 15.1 & 33.1 & 34.2 \\
\hline \multicolumn{5}{|l|}{ METs-min/week, $M(S D)$} \\
\hline Active travel & $18.40(84.51)$ & $1,941.90(1,494.56)$ & $25.46(98.05)$ & $1,668.62(1,189.01)$ \\
\hline LTPA & $57.56(141.67)$ & $50.95(140.57)$ & $1,660.11(1,227.38)$ & $2,099.45(1,578.88)$ \\
\hline Work-related activity & $727.97(2,141.47)$ & $1,119.87(2,715.87)$ & $1,045.94(2,742.60)$ & $1,972.70(3,488.11)$ \\
\hline \multicolumn{5}{|l|}{ Duration/day, $M(S D)$} \\
\hline Active travel & $1.59(7.38)$ & $86.10(62.14)$ & $2.21(8.63)$ & $79.32(52.18)$ \\
\hline LTPA & $6.66(15.25)$ & $6.54(19.23)$ & $90.52(60.46)$ & $104(67.47)$ \\
\hline Work-related activity & $39.25(98.63)$ & $51.55(112.47)$ & $53.93(110.37)$ & $93.30(136.30)$ \\
\hline
\end{tabular}

Note. LTPA = leisure-time physical activity; MET = metabolic equivalent of task.

Questionnaire. The participants were asked about their engagement in LTPA and active travel in a typical week, along with dose (frequency in days/week and average duration/session). Moderate-intensity LTPA was assessed through the following question: "In a typical week, do you do any moderate intensity sports, fitness, or recreational activities that cause a small increase in breathing or heart rate such as brisk walking, bicycling, swimming, or volleyball for at least 10 minutes continuously?" The participants reported on the dose (i.e., frequency days/week, average duration per session). Vigorousintensity LTPA was assessed in a similar manner through the following question: "In a typical week, do you do any vigorous intensity sports, fitness, or recreational activities that cause a large increase in breathing or heart rate such as running or basketball, for at least 10 minutes continuously?" As a measure of active travel, the participants reported on the extent to which they engaged in moderateintensity walking or cycling as a means of transport (>10 min), along with dose (i.e., frequency in days/week, average duration). The participants also reported on their work-related physical activity in a similar manner, which was included as a covariate in all analyses. Metabolic equivalent of task (MET) values were assigned to each of the activities, using the NHANES guidelines. Moderate-intensity activity was assigned an MET value of 4.0 (i.e., active travel and moderate-intensity LTPA), and vigorous-intensity activity was assigned an MET value of 8.0 (i.e., vigorous-intensity LTPA). Although active travel and LTPA have some common activities, such as walking and bicycling, the questions defined the context in which these activities are to be performed to assist respondents' classification. For example, walking to the grocery store would be active travel, but walking on the running track in the gym would be LTPA. Accordingly, the METs assigned to them depended on whether they were reported as moderate or vigorous LTPA or active travel by the respondent. After assigning the corresponding MET values, a composite score reflecting the total activity per week was created for LTPA and active travel each, by multiplying the assigned MET with dose (i.e., frequency and duration). For example, an individual engaging in moderate-intensity physical activity (4 METs) for $20 \mathrm{~min}, 4$ times/week would get a score of $4 \times 20 \times 4=320 \mathrm{MET} /$ week. Four groups were created based on whether the participants met the physical activity guidelines (600 MET/week) through the respective activities. About $600 \mathrm{MET} /$ week was chosen as the cutoff because it aligns with the Centre for Disease Control's recommendation for physical activity (i.e., $150 \mathrm{~min}$ of moderate-intensity activity/week). Those above the cutoff were considered to be meeting the guidelines with respect to the particular activity. This method resulted in four groups: not meeting physical activity guidelines $(n=1,790)$, meeting guidelines only through active travel $(n=210)$, meeting guidelines only through LTPA $(n=579)$, and meeting guidelines through both active travel and LTPA $(n=123)$.

Cognition. The Digit Symbol Substitution Test (DSST) is a module from the Wechsler and Psychological Corporation (1997) Adult Intelligence Scale and generally is used as a measure of processing speed, though it requires sustained attention, working memory, and executive function. The task involves matching a series of numbers with symbols based on a code key containing symbols assigned to nine numbers (0-9). A sheet containing 133 numbers ranging from 0 to 9 , arranged in a random order, with blank boxes next to them, is provided. The task requires that the corresponding symbol be drawn in the blank box by using the code key, 
for as many numbers as possible within 2 min, without skipping a box. The total score is the number of correct substitutions (maximum possible score $=133)$. Higher scores indicate a better performance.

Animal Fluency Test (AFT) is a measure of categorical verbal fluency, which is a component of executive function (Strauss, Sherman, \& Spreen, 2006). It has been shown to differentiate among those who have cognitive impairment or dementia in the older population (Sebaldt et al., 2009). The task requires naming as many animals as possible in $1 \mathrm{~min}$. Plural forms are not counted as a separate item. To familiarize participants with the task, a practice trail is provided, whereby individuals name three items of clothing. The total score is the number of animals named correctly in $1 \mathrm{~min}$. Higher scores indicate better performance.

Delayed Recall was assessed via Consortium to Establish a Registry for Alzheimer's Disease (CERAD) World Learning subtest. Delayed recall is thought to be the best measure among other CERAD tasks for differentiating between healthy older adults and individuals with dementia (Welsh, Butters, Hughes, Mohs, \& Heyman, 1991). The task requires participants to recall as many words as possible, in no particular order, from a list of 10 words that is presented to them $8-10 \mathrm{~min}$ prior to the recall task. The list of 10 words is presented one at a time on a computer screen (for those with visual impairment, the words are read aloud). While assessing this measure in NHANES, during the 8- to 10-min gap provided between the presentation of the words and the recall task, DSST and AFT were administered, thus, preventing the participants from rehearsing the words in the list. The total score is the number of words correctly recalled (maximum possible score $=10$ ). Higher scores indicate better performance.

\section{Data Analysis}

The data were weighted using appropriate weights (for the cluster, strata, and sample) provided in the data set, per the instructions provided on the NHANES website, in order to get an estimate of a nationally representative sample. NHANES uses a complex, multistage, probability sampling design. In order to account for this complex survey design, each person is assigned a weight. This weight is based on a number of steps. First, the probability of demographics are calculated (e.g., person's city, block, household). Then, the weight takes into account nonresponse and noncoverage, and whether the person is selected for a subsample in the study. This is followed by poststratification adjustment so that the sample matches the U.S. 2000 Census population estimates. Detailed information is provided in the tutorial on the NHANES website (https:// www.cdc.gov/nchs/tutorials/environmental/orientation/sample_ design/). The complex samples procedure in SPSS (IBM SPSS Statistics for Windows, version 25.0; IBM Corp., Armonk, NY) was used to create a weighted analysis file, which applies the sample weight while estimating the sampling error provided by variables in the NHANES data set (accounting for strata and primary sampling units). General Linear Modeling was then used to conduct univariate analysis of covariance on the weighted data. Data were checked for violations of assumptions of analysis of variance. Dependent variables were normally distributed, as examined by histograms and P-P plots. Data were winsorized for univariate outliers, which were values above $2.5 \mathrm{SDs}$, which is a robust method to deal with extreme values (Erceg-Hurn \& Mirosevich, 2008). Specifically, the reported duration of each of the physical activities, the calculated total physical activity scores, and the scores on the cognitive variables were checked for univariate outliers and were winsorized if outliers were present. Except for animal fluency and delayed recall scores, the rest of the variables were winsorized due to the presence of outliers. Less than $10 \%$ of the total cases required winsorization for any of the variables. The maximum cutoffs used were 240 min for duration of active travel on a typical day, 180 min for duration of vigorous recreational activity on a typical day, 230 min for duration of moderate recreational activity on a typical day, 2,208 MET-min for total active travel score, 3,000 MET-min for total recreational activity score, and a score of 94 for DSST. Based on prior research showing that cognitive benefits of exercise occur after $20 \mathrm{~min}$ of exercise at minimum (Brisswalter, Collardeau, \& René, 2002), the respondents who reported engaging in $<20$ min of average active travel or LTPA were coded as inactive. Among all respondents $(n=3,472)$, only 2,702 respondents had complete data for the variables included in the analysis. An univariate analysis of covariance was used to determine the relationship between DSST, AFT, and DR and the four groups of physical activity. Covariates included sex, race, education (all of which were dummy coded), age, and body mass index. Pairwise comparisons were conducted using simple contrasts with Bonferroni adjustment. Effect sizes using Cohen's $d$ were calculated for multiple comparisons. However, these must be interpreted with caution, as they may not be directly comparable with other samples due to the complex survey design and weighting of the data.

\section{Results}

The mean age of the sample was $69.95(S D=6.94), 51.6 \%$ female, $47.6 \%$ non-Hispanic White, and $23.2 \%$ having at least a college graduate degree. Among the $10 \%$ of the sample that had data on occupation, a fifth $(\sim 20 \%)$ of them reported working more than $35 \mathrm{hr} /$ week at present. Demographic characteristics across the four groups of physical activity are shown in Table 1 . The means for each of the groups on each dependent variable are shown in Table 2.

\section{Digit Symbol Substitution Test}

Univariate analysis revealed a significant group effect for DSST, $F(3,30)=15.82, p<.001$, after controlling for covariates. Age, gender, race, education, and work-related activity were significant predictors of DSST scores. The overall model explained $37.1 \%$ of the variance in DSST scores. The inactive group $(M=50.49$

Table 2 Mean Values for Each Group Across Each of the Cognition Variables, Adjusted for Covariates

\begin{tabular}{|c|c|c|c|c|}
\hline Cognitive outcome & Inactive & Active travel & LTPA & Both activities \\
\hline Digit Symbol Substitution Test & $50.41(0.50)^{\mathrm{a}, \mathrm{b}}$ & $50.96(1.35)$ & $56.07(0.68)^{\mathrm{a}}$ & $53.83(1.05)^{\mathrm{b}}$ \\
\hline Animal Fluency Test & $17.42(0.11)^{\mathrm{a}, \mathrm{b}}$ & $18.04(0.55)$ & $19.29(0.25)^{\mathrm{a}}$ & $19.48(0.76)^{\mathrm{b}}$ \\
\hline Delayed Recall & $6.08(0.08)^{\mathrm{a}}$ & $6.58(0.24)$ & $6.54(0.11)^{\mathrm{a}}$ & $6.10(0.21)$ \\
\hline
\end{tabular}

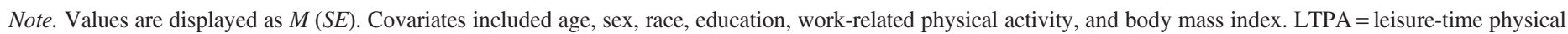
activity. Significant letters $(\mathrm{a}, \mathrm{b})$ indicate that groups are different from each other at $\alpha=.05$. 
$[S D=20.27])$ and active travel group $(M=50.82[S D=20.09])$ were not statistically different from each other. The DSST scores were, however, significantly higher among those meeting the physical activity guidelines via LTPA $(M=56.00[S D=16.03])$ rather than those who were inactive $(M=50.49$ [SD $=20.27]$, $p<.001, d=0.30)$. Those engaging in both types of physical activity (active travel + LTPA; $M=53.58[S D=11.76]$ ) were also significantly different from the inactive group $(M=50.49$ $[S D=20.27], p<.05, d=0.18)$.

\section{Animal Fluency Test}

Univariate analysis revealed a significant group effect for AFT, $F(3,30)=17.44, p<.001$. Age, race, and education were significant predictors of AFT scores. The overall model explained $23.6 \%$ of the variance in AFT scores. Similar to DSST, the inactive $(M=$ $17.46[S D=4.56])$ and active travel groups $(M=17.98[S D=8.01])$ were not statistically different from each other. Those engaging in LTPA $(M=19.26[S D=6.01], p<.001, d=0.34)$ and both types of activities $(M=19.40[S D=8.55], p<.05, d=0.28)$ performed significantly better than those in the inactive group $(M=17.46$ $[S D=4.56])$.

\section{Delayed Recall}

Univariate analysis revealed a significant group effect for DR, $F(3,30)=10.24, p<.001$. Age, gender, race, and education were significant predictors of DR scores. Those who engaged in LTPA $(M=6.54[S D=2.74])$ scored better than those in the inactive group $(M=6.09[S D=3.67], p<.001, d=0.14)$. There were no significant differences between the other groups.

\section{Discussion}

In our analysis, we found that individuals engaging in at least 600 METs of LTPA have significantly higher scores on tests of processing speed, verbal fluency, and delayed recall than those who are inactive (PA lesser than $600 \mathrm{MET} /$ week), with the magnitude of difference ranging between small and medium. There was no significant difference between active travelers and those who were inactive on any of the cognition variables. Individuals who engaged in both LTPA as well as active travel performed significantly and slightly better on the tests of verbal fluency and processing speed, but not on delayed recall, compared with those who were inactive. This suggests that engaging in active travel over and above LTPA may not have a significant influence on memory. Engaging in LTPA appears to be the common thread underlying benefits for executive function and memory. There is a possibility that respondents may not have correctly reported their physical activity. Even though the order of the questions assessing physical activity ensured that active travel was assessed prior to LTPA, there is a possibility that respondents may have incorrectly categorized their physical activity (e.g., walking to the park may have been reported as LTPA).

Our findings are similar to those found by Rovio et al. (2007), whereby active travel in late midlife (mean age $=50$ years) was not significantly associated with the risk of dementia later in life, when controlled for demographic factors and LTPA. Apart from this study, most studies examining the association between active travel and cognition have been conducted among children and adolescents, with mixed evidence. For example, Van Dijk et al. (2014) found that active travel to school was unrelated to cognitive function among adolescents, but girls who engaged in active commuting showed a significant improvement on the $\mathrm{d} 2$ test of attention, but this effect was not seen in boys. Other studies have reported that cognitive improvements are associated with active travel (López-Vicente et al., 2016; Martínez-Gómez et al., 2011). For example, in a study among adolescents, girls who engaged in active travel scored significantly higher on verbal, numerical, reasoning, and overall cognitive ability compared with those who did not engage in active traveling, but a similar effect was not observed among boys (Martínez-Gómez et al., 2011). It may be the case that the relationship between active travel and cognition varies between genders. Furthermore, the relationships found may not exist in a similar manner among older adults. Provided that few studies have previously looked at this relationship exclusively among older adults, future research should not only explore the association between active travel and cognition among older adults, but also examine whether gender has a moderating effect.

A potential reason for the nonsignificant association of active travel with cognition in this analysis may be the insufficient duration of the bout of active travel. While the mean duration of active travel per day was $86 \min (S D=62.14)$, it may not necessarily represent sustained activity. The active travel assessment within NHANES did not require participants to mention the number of bouts of active travel throughout the day. Thus, it is unclear whether the selfreported minutes of activity account for multiple bouts of active travel during the day (traveling to and from places), whose exact duration is not known. Indeed, when Rovio et al. (2007) found no association between active travel and dementia risk, the number of bouts that may have constituted the total amount of daily active travel was not measured. In comparison, studies that found significant improvements in cognition from active travel have reported the duration of a single bout of active travel (e.g., traveling to the school). For example, in these studies, the mean duration of the single bout of active travel was $46 \mathrm{~min} /$ day (mean min/week = 230.80, $S D=94.75$; Van Dijk et al., 2014), or $>15 \mathrm{~min} /$ day (Martínez-Gómez et al., 2011), or between 30 and $60 \mathrm{~min} /$ day (García-Hermoso et al., 2017). Furthermore, these studies targeted adolescents, and it is possible that older adults need a higher dosage of physical activity in order to achieve cognitive benefits.

We found that LTPA was associated with improvements in executive function and memory, a finding that is supported among older adults (Bherer et al., 2013; Kramer \& Erickson, 2007). Evidence for this comes from a variety of studies with older adults (aged 60 years and older) involving cross-sectional surveys (Loprinzi, Edwards, Crush, Ikuta, \& Del Arco, 2018), prospective cohort designs (Weuve et al., 2004), acute interventions (Barella et al., 2010; Córdova, Silva, Moraes, Simões, \& Nóbrega, 2009; Kamijo et al., 2009), and randomized controlled trials (Albinet, Boucard, Bouquet, \& Audiffren, 2010; Kramer, Colcombe, McAuley, Scalf, \& Erickson, 2005; Smiley-Oyen, Lowry, Francois, Kohut, \& Ekkekakis, 2008). For example, Smiley-Oyen et al. (2008) conducted an randomized controlled trial comparing a group doing aerobic exercises with various gym equipment with a flexibility group for 10 months, and found that the aerobic exercise group improved executive function among previously inactive older adults with a mean age of 70 years.

Although active travel and LTPA involve physical activity, LTPA has additional features that may enhance cognitive function. For example, it has been hypothesized that LTPAs that involve complex movements or mind-body activities, including martial arts, yoga, sports, or dancing, may be more beneficial for cognition compared with activities that require less neuromotor coordination, such as walking (Diamond \& Ling, 2016). It is likely that the older adults in our sample may be engaging in such activities as a part 
of their LTPA. These activities may also result in a greater variety of physical benefits due to their complex nature. Furthermore, certain types of LTPA, such as group fitness classes (e.g., Silver Sneakers) and "masters level" sports participation may provide more opportunities for social interaction compared with active travel (a solo activity). Altogether, LTPA may result in benefits to cognition through more sources than active travel. Apart from this, the adults in this sample reported engaging in moderate or vigorous intensity LTPA for about $60 \mathrm{~min} / \mathrm{session}, 3-4$ times a week. While this mean duration is similar to the duration of active travel, the duration for LTPA may be more likely to represent elongated bouts of activity.

We found that those engaging in both types of activities, i.e., LTPA and active travel, performed significantly better than those who were inactive, on DSST and AFT, but not on DR. The effects of physical activity on memory are time dependent. Research has shown that effects on memory may be most pronounced when memory is tested immediately after the physical activity (Frith, Sng, \& Loprinzi, 2017; Roig et al., 2016; Sng, Frith, \& Loprinzi, 2018). In this study, the physical activity engagement of the participants before their cognitive testing session is unclear, and we cannot determine when the respondents performed their last bout of physical activity and its duration. This time-sensitive relationship may have affected their performance on the memory task, creating uncontrolled variability. Another reason could be the potential dose-response relationship of the physical activity duration to cognition. This is in line with prior research, which has found mixed evidence for the dose-response relationship between physical activity and cognition, with studies finding either a linear (Xu et al., 2011), inverted-U (Loprinzi et al., 2018), or no dose-response relationship (Sanders, Hortobágyi, la Bastidevan Gemert, van der Zee, \& van Heuvelen, 2019). For example, a recent meta-analysis among older adults showed that, whereas exercise had a small positive impact on cognition, exercise duration, frequency, or program length were not significant predictors of the dose-response relationship with cognition (Sanders et al., 2019).

This study has several limitations. Because the analysis is cross-sectional, directionality between active travel or LTPA and cognition is unknown. It is possible that those who have higher cognitive functioning engage in higher amounts of active travel or LTPA, rather than the inverse. The finding that active travel was not associated with enhanced cognitive functioning needs to be further explored through randomized controlled trials. Since the data are self-reported, they may be susceptible to recall bias, as well as social desirability effects. In order to portray themselves as being physically active, the participants may overreport their activity levels. The participants may have overreported or underreported their travel, as well as their LTPA, which may have resulted in a nonsignificant association between active travel and cognition. Moreover, this association needs to be further examined with different levels of duration and intensity of active travel, using objective measures, as physical activity has a dose-response relationship with some aspects of cognitive functioning.

In conclusion, the present study supported our hypothesis about LTPA and cognition by showing that engaging in LTPA was associated with a small increase in cognitive performance. With respect to the relationship between active travel and cognition, our study found no association with improvements in cognition after adjusting for other confounding variables. Given the limitations of the present study, future studies should systematically examine if and when active travel influences cognitive functioning.

\section{References}

Abbott, R.D., White, L.R., Ross, G.W., Masaki, K.H., Curb, J.D., \& Petrovitch, H. (2004). Walking and dementia in physically capable elderly men. Journal of American Medical Association, 292(12), 1447-1453. doi:10.1001/jama.292.12.1447

Albinet, C.T., Boucard, G., Bouquet, C.A., \& Audiffren, M. (2010). Increased heart rate variability and executive performance after aerobic training in the elderly. European Journal of Applied Physiology, 109(4), 617-624. PubMed ID: 20186426 doi:10.1007/s00421010-1393-y

Angevaren, M., Vanhees, L., Wendel-Vos, W., Verhaar, H.J., Aufdemkampe, G., Aleman, A., \& Verschuren, W.M. (2007). Intensity, but not duration, of physical activities is related to cognitive function. European Journal of Cardiovascular Prevention and Rehabilitation, 14(6), 825-830. PubMed ID: 18043306 doi:10.1097/HJR.0b013e3282ef995b

Barella, L.A., Etnier, J.L., \& Chang, Y.-K. (2010). The immediate and delayed effects of an acute bout of exercise on cognitive performance of healthy older adults. Journal of Aging and Physical Activity, 18(1), 87-98. PubMed ID: 20181996 doi:10.1123/japa.18.1.87

Bherer, L., Erickson, K.I., \& Liu-Ambrose, T. (2013). A review of the effects of physical activity and exercise on cognitive and brain functions in older adults. Journal of Aging Research, 2013, 657508. PubMed ID: 24102028 doi:10.1155/2013/657508

Brisswalter, J., Collardeau, M., \& René, A. (2002). Effects of acute physical exercise characteristics on cognitive performance. Sports Medicine, 32(9), 555-566. PubMed ID: 12096929 doi:10.2165/ 00007256-200232090-00002

Cerin, E., Nathan, A., Van Cauwenberg, J., Barnett, D.W., \& Barnett, A. (2017). The neighbourhood physical environment and active travel in older adults: A systematic review and meta-analysis. International Journal of Behavioral Nutrition and Physical Activity, 14(1), 15. PubMed ID: 28166790 doi:10.1186/s12966-017-0471-5

Chudyk, A.M., Winters, M., Moniruzzaman, M., Ashe, M.C., Gould, J.S., \& McKay, H. (2015). Destinations matter: The association between where older adults live and their travel behavior. Journal of Transport \& Health, 2(1), 50-57. PubMed ID: 27104147 doi:10.1016/j.jth. 2014.09.008

Córdova, C., Silva, V., Moraes, C.F., Simões, H.G., \& Nóbrega, O.T. (2009). Acute exercise performed close to the anaerobic threshold improves cognitive performance in elderly females. Brazilian Journal of Medical and Biological Research, 42(5), 458-464. doi:10.1590/ S0100-879X2009000500010

Diamond, A., \& Ling, D.S. (2016). Conclusions about interventions, programs, and approaches for improving executive functions that appear justified and those that, despite much hype, do not. Developmental Cognitive Neuroscience, 18, 34-48. PubMed ID: 26749076 doi:10.1016/j.den.2015.11.005

Erceg-Hurn, D.M., \& Mirosevich, V.M. (2008). Modern robust statistical methods: An easy way to maximize the accuracy and power of your research. American Psychologist, 63(7), 591-601. PubMed ID: 18855490 doi:10.1037/0003-066X.63.7.591

Frith, E., Sng, E., \& Loprinzi, P.D. (2017). Randomized controlled trial evaluating the temporal effects of high-intensity exercise on learning, short-term and long-term memory, and prospective memory. European Journal of Neuroscience, 46(10), 2557-2564. PubMed ID: 28922507 doi:10.1111/ejn.13719

Gallotta, M.C., Guidetti, L., Franciosi, E., Emerenziani, G.P., Bonavolonta, V., \& Baldari, C. (2012). Effects of varying type of exertion on children's attention capacity. Medicine and Science in Sports and Exercise, 44(3), 550-555. PubMed ID: 21814148 doi:10.1249/MSS. Ob013e3182305552 
García-Hermoso, A., Saavedra, J.M., Olloquequi, J., \& Ramírez-Vélez, R. (2017). Associations between the duration of active commuting to school and academic achievement in rural Chilean adolescents. Environmental Health and Preventive Medicine, 22(1), 31. doi:10. 1186/s12199-017-0628-5

Gligoroska, J.P., \& Manchevska, S. (2012). The effect of physical activity on cognition-physiological mechanisms. Materia Socio-Medica, 24(3), 198. PubMed ID: 23678325 doi:10.5455/msm.2012.24. 198-202

Hillman, C.H., Erickson, K.I., \& Kramer, A.F. (2008). Be smart, exercise your heart: Exercise effects on brain and cognition. Nature Reviews Neuroscience, 9(1), 58-65. PubMed ID: 18094706 doi:10.1038/ nrn2298

Kamijo, K., \& Abe, R. (2019). Aftereffects of cognitively demanding acute aerobic exercise on working memory. Medicine and Science in Sports and Exercise, 51(1), 153-159. PubMed ID: 30153193 doi:10.1249/ MSS.0000000000001763

Kamijo, K., Hayashi, Y., Sakai, T., Yahiro, T., Tanaka, K., \& Nishihira, Y. (2009). Acute effects of aerobic exercise on cognitive function in older adults. Journals of Gerontology: Series B, 64(3), 356-363. doi:10.1093/geronb/gbp030

King, A.C., Sallis, J.F., Frank, L.D., Saelens, B.E., Cain, K., Conway, T.L., . . . Kerr, J. (2011). Aging in neighborhoods differing in walkability and income: Associations with physical activity and obesity in older adults. Social Science \& Medicine, 73(10), 15251533. PubMed ID: 21975025 doi:10.1016/j.socscimed.2011.08.032

Kramer, A.F., Colcombe, S.J., McAuley, E., Scalf, P.E., \& Erickson, K.I. (2005). Fitness, aging and neurocognitive function. Neurobiology of Aging, 26(1), 124-127. doi:10.1016/j.neurobiolaging.2005.09.009

Kramer, A.F., \& Erickson, K.I. (2007). Capitalizing on cortical plasticity: Influence of physical activity on cognition and brain function. Trends in Cognitive Sciences, 11(8), 342-348. PubMed ID: 17629545 doi:10.1016/j.tics.2007.06.009

Laverty, A.A., Palladino, R., Lee, J.T., \& Millett, C. (2015). Associations between active travel and weight, blood pressure and diabetes in six middle income countries: A cross-sectional study in older adults. International Journal of Behavioral Nutrition and Physical Activity, 12(1), 65. doi:10.1186/s12966-015-0223-3

López-Vicente, M., Forns, J., Esnaola, M., Suades-González, E., ÁlvarezPedrerol, M., Robinson, O., . . . Sunyer, J. (2016). Physical activity and cognitive trajectories in schoolchildren. Pediatric Exercise Science, 28(3), 431-438. doi:10.1123/pes.2015-0157

Loprinzi, P.D., Edwards, M.K., Crush, E., Ikuta, T., \& Del Arco, A. (2018). Dose-response association between physical activity and cognitive function in a national sample of older adults. American Journal of Health Promotion, 32(3), 554-560. PubMed ID: 29214828 doi:10.1177/0890117116689732

Martínez-Gómez, D., Ruiz, J.R., Gómez-Martínez, S., Chillón, P., ReyLópez, J.P., Díaz, L.E., . . Marcos, A. (2011). Active commuting to school and cognitive performance in adolescents: The AVENA study. Archives of Pediatrics \& Adolescent Medicine, 165(4), 300-305. doi:10.1001/archpediatrics.2010.244

Michael, Y.L., Green, M.K., \& Farquhar, S.A. (2006). Neighborhood design and active aging. Health \& Place, 12(4), 734-740. PubMed ID: 16159710 doi:10.1016/j.healthplace.2005.08.002

Ng, T.P., Nyunt, M.S.Z., Shuvo, F.K., Eng, J.Y., Yap, K.B., Hee, L.M., . . Scherer, S. (2018). The neighborhood built environment and cognitive function of older persons: Results from the Singapore longitudinal ageing study. Gerontology, 64(2), 149-156. PubMed ID: 28910813 doi:10.1159/000480080

Ogilvie, D., Foster, C.E., Rothnie, H., Cavill, N., Hamilton, V., Fitzsimons, C.F., \& Mutrie, N. (2007). Interventions to promote walking: Systematic review. The British Medical Journal, 334(7605), 1204. doi:10.1136/bmj.39198.722720.BE

O'Leary, K.C., Pontifex, M.B., Scudder, M.R., Brown, M.L., \& Hillman, C.H. (2011). The effects of single bouts of aerobic exercise, exergaming, and videogame play on cognitive control. Clinical Neurophysiology, 122(8), 1518-1525. doi:10.1016/j.clinph.2011.01.049

Oxley, J. (2015). Understanding travel patterns to support safe active transport for older adults. Journal of Transport \& Health, 2(1), 79-85. doi:10.1016/j.jth.2014.09.016

Pratt, M., Macera, C.A., Sallis, J.F., O’Donnell, M., \& Frank, L.D. (2004). Economic interventions to promote physical activity: Application of the SLOTH model. American Journal of Preventive Medicine, 27(3), 136-145. doi:10.1016/j.amepre.2004.06.015

Richards, M., Hardy, R., \& Wadsworth, M.E. (2003). Does active leisure protect cognition? Evidence from a national birth cohort. Social Science \& Medicine, 56(4), 785-792. PubMed ID: 12560011 doi:10.1016/S0277-9536(02)00075-8

Roig, M., Thomas, R., Mang, C.S., Snow, N.J., Ostadan, F., Boyd, L.A., \& Lundbye-Jensen, J. (2016). Time-dependent effects of cardiovascular exercise on memory. Exercise and Sport Sciences Reviews, 44(2), 81-88. PubMed ID: 26872291 doi:10.1249/JES.0000000000000078

Rovio, S., Kåreholt, I., Helkala, E.-L., Viitanen, M., Winblad, B., Tuomilehto, J., . . . Kivipelto, M. (2005). Leisure-time physical activity at midlife and the risk of dementia and Alzheimer's disease. The Lancet Neurology, 4(11), 705-711. PubMed ID: 16239176 doi:10.1016/S1474-4422(05)70198-8

Rovio, S., Kåreholt, I., Viitanen, M., Winblad, B., Tuomilehto, J., Soininen, H., ... Kivipelto, M. (2007). Work-related physical activity and the risk of dementia and Alzheimer's disease. International Journal of Geriatric Psychiatry, 22(9), 874-882. PubMed ID: 17721898 doi:10.1002/gps.1755

Sanchez-Lopez, J., Silva-Pereyra, J., Fernández, T., Alatorre-Cruz, G.C., Castro-Chavira, S.A., González-López, M., \& Sánchez-Moguel, S.M. (2018). High levels of incidental physical activity are positively associated with cognition and EEG activity in aging. PLoS One, 13(1), e0191561. PubMed ID: 29370215 doi:10.1371/journal.pone. 0191561

Sanders, L.M.J., Hortobágyi, T., la Bastide-van Gemert, S., van der Zee, E.A., \& van Heuvelen, M.J.G. (2019). Dose-response relationship between exercise and cognitive function in older adults with and without cognitive impairment: A systematic review and metaanalysis. PLoS One, 14(1), e0210036. PubMed ID: 30629631 doi:10. 1371/journal.pone.0210036

Scherder, E., Scherder, R., Verburgh, L., Königs, M., Blom, M., Kramer, A.F., \& Eggermont, L. (2014). Executive functions of sedentary elderly may benefit from walking: A systematic review and meta-analysis. The American Journal of Geriatric Psychiatry, 22(8), 782-791. PubMed ID: 23636004 doi:10.1016/j.jagp.2012. 12.026

Sebaldt, R., Dalziel, W., Massoud, F., Tanguay, A., Ward, R., Thabane, L., ... Lescrauwaet, B. (2009). Detection of cognitive impairment and dementia using the animal fluency test: The DECIDE study. Canadian Journal of Neurological Sciences, 36(5), 599-604. PubMed ID: 19831129 doi:10.1017/S0317167100008106

Smiley-Oyen, A.L., Lowry, K.A., Francois, S.J., Kohut, M.L., \& Ekkekakis, P. (2008). Exercise, fitness, and neurocognitive function in older adults: The "selective improvement" and "cardiovascular fitness" hypotheses. Annals of Behavioral Medicine, 36(3), 280-291. PubMed ID: 18825471 doi:10.1007/s12160-008-9064-5

Smith, G.C., \& Sylvestre, G.M. (2001). Determinants of the travel behavior of the suburban elderly. Growth and Change, 32(3), 395-412. doi:10.1111/0017-4815.00165 
Sng, E., Frith, E., \& Loprinzi, P.D. (2018). Temporal effects of acute walking exercise on learning and memory function. American Journal of Health Promotion, 32(7), 1518-1525. PubMed ID: 29284283 doi:10.1177/0890117117749476

Strauss, E., Sherman, E., \& Spreen, O. (2006). A compendium of neuropsychological tests: Administration, norms, and commentary (3rd ed.). New York, NY: Oxford University Press.

Vancampfort, D., Smith, L., Stubbs, B., Swinnen, N., Firth, J., Schuch, F.B., \& Koyanagi, A. (2018). Associations between active travel and physical multi-morbidity in six low- and middle-income countries among community-dwelling older adults: A cross-sectional study. PLoS One, 13(8), e0203277. PubMed ID: 30161211 doi:10.1371/ journal.pone.0203277

Van Dijk, M.L., De Groot, R.H., Van Acker, F., Savelberg, H.H., \& Kirschner, P.A. (2014). Active commuting to school, cognitive performance, and academic achievement: An observational study in Dutch adolescents using accelerometers. BMC Public Health, 14(1), 799. doi:10.1186/1471-2458-14-799

Wechsler, D., \& Psychological Corporation. (1997). Wais-III: Administration and scoring manual: Wechsler adult intelligence scale (3rd ed.). San Antonio, TX: Psychological Corporation.

Welsh, K., Butters, N., Hughes, J., Mohs, R., \& Heyman, A. (1991). Detection of abnormal memory decline in mild cases of Alzheimer's disease using CERAD neuropsychological measures. Archives of Neurology, 48(3), 278-281. PubMed ID: 2001185 doi:10.1001/ archneur.1991.00530150046016

Weuve, J., Kang, J.H., Manson, J.E., Breteler, M.M., Ware, J.H., \& Grodstein, F. (2004). Physical activity, including walking, and cognitive function in older women. Journal of American Medical Association, 292(12), 1454-1461. doi:10.1001/jama. 292.12.1454

Xu, L., Jiang, C.Q., Lam, T.H., Zhang, W.S., Thomas, G.N., \& Cheng, K.K. (2011). Dose-response relation between physical activity and cognitive function: Guangzhou Biobank Cohort study. Annals of Epidemiology, 21(11), 857-863. PubMed ID: 21784658 doi:10.1016/ j.annepidem.2011.06.002

Yaffe, K., Barnes, D., Nevitt, M., Lui, L.-Y., \& Covinsky, K. (2001). A prospective study of physical activity and cognitive decline in elderly women: Women who walk. Archives of Internal Medicine, 161(14), 1703-1708. PubMed ID: 11485502 doi:10.1001/archinte.161.14. 1703

Yang, Y., Roux, A.V.D., \& Bingham, C.R. (2011). Variability and seasonality of active transportation in USA: Evidence from the 2001 NHTS. International Journal of Behavioral Nutrition and Physical Activity, 8(1), 96. doi:10.1186/1479-5868-8-96 